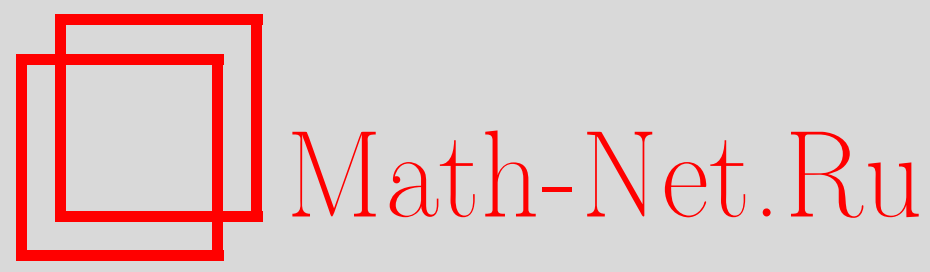

Н. Г. Мощевитин, Дифференциальные уравнения с почти и условно периодическими коэффициентами: возвращаемость и приводимость, Матем. заметки, 1998, том 64, выпуск $2,229-237$

DOI: https://doi.org/10.4213/mzm1391

Использование Общероссийского математического портала Math-Net.Ru подразумевает, что вы прочитали и согласны с пользовательским соглашением http://www . mathnet.ru/rus/agreement

Параметры загрузки:

IP : 54.164 .48 .24

26 апреля 2023 г., 15:06:02

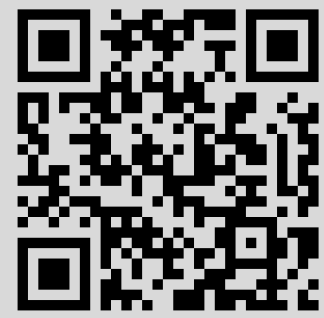




\section{ДИФФЕРЕНЦИАЛЬНЫЕ УРАВНЕНИЯ С ПОЧТИ И УСЛОВНО ПЕРИОДИЧЕСКИМИ КОЭФФИЦИЕНТАМИ: ВОЗВРАШАЕМОСТЬ И ПРИВОДИМОСТЬ}

\section{Н. Г. Мощевитин}

Доказьвается возвращаемость индивидуальной траектории неавтономного линейного дифференциального уравнения на компактной группе Ли в случае почти и условно периодической зависимости от времени правой части. Изучается связь задачи о возврашаемости с вопросами приводимости.

Библиография: 19 названий.

1. Введение. Пусть $v(x)$ - векторное поле на компактном гладком многообразии $M$ такое, что $\operatorname{div} v \equiv 0$. Как известно, в этом случае дифференциальное уравнение $\dot{x}=v(x)$ сохраняет фазовый объем и справедлива теорема Пуанкаре о возвращаемости [1]: почти всякая траектория уравнения $\dot{x}=v(x)$ бесконечно много раз возврашается к своему начальному условию. Эта теорема допускает небольшое количественное уточнение [2]. Обозначим через $|\cdot|$ какое-либо расстояние на $M$, через $x\left(t, x_{0}\right)$ траекторию с начальным условием $x_{0}$. Тогда существует положительное $\delta=\delta(M, v)$ такое, что для почти всякого (в смысле меры Лебега) $x_{0} \in M$ найдется последовательность моментов времени $t_{\nu} \rightarrow+\infty$, для которой вьполнено $\left|x\left(t_{\nu}, x_{0}\right)-x_{0}\right|<t_{\nu}^{-\delta}$. Последний результат является метрическим, так как связан с поведением почти всех траекторий.

В настоящей работе получены результаты о возврашаемости всех траекторий неавтономного уравнения

$$
\dot{X}=A(t) X,
$$

где $X \in \mathrm{SO}(n)$ и $A(t) \subset \mathrm{so}(n)$ - гладкая кососимметрическая матрица, почти или условно периодически зависящая от времени $t$.

В случае условно периодической зависимости уравнение (1) может быть записано в расширенном виде

$$
\dot{X}=D(\varphi) X, \quad \dot{\varphi}=\omega, \quad X \in \mathrm{SO}(n), \quad \varphi \in \mathbb{T}^{s}
$$

$\left(\omega=\left(\omega_{1}, \ldots, \omega_{s}\right)-\right.$ базис частот условно периодической кососимметрической матрицы $\left.A(t)=D\left(\omega t+\varphi_{0}\right)\right)$. Последнее уравнение является автономным (но уже нелинейньп) на компактной групе $\mathrm{SO}(n) \times \mathbb{T}^{s}$, и по теореме Пуанкаре почти всякая траектория этого

Работа выполнена при финансовой поддержке Российского фонда фундаментальных исследований, гранты № 96-01-00378 и № 96-15-96072. 
уравнения возвращается. Тем не менее, теорема Пуанкаре ничего не говорит о возвращаемости каждой траектории.

Отметим, что в случае периодической зависимости от $t$ матрицы $A(t)$ уравнение (1) приводимо и, следовательно, каждая его траектория возвращается, причем регулярно.

Ниже все результаты формулируются для линейного неавтономного уравнения на группе $\mathrm{SO}(n)$. Однако, они остаются справедливыми и для любого левоинвариантного почти или условно периодического векторного поля на любой компактной связной группе Ли, поскольку последнюю всегда можно вложить в $\mathrm{SO}(n)$ при достаточно большом $n$.

Отметим, что идеи доказательств почерпнуты из некоторых элементарных методов в задачах теории чисел, связанных с итерационными процессами [3]-[5].

2. Возвращаемость. Зафиксируем некоторую норму в пространстве всех матриц и обозначим через $|\cdot|$.

Теорема 1. Пусть непрерывная матрица $A(t) \subset \operatorname{so}(n)$ удовлетворяет условию

$$
\forall \varepsilon>0 \quad \forall T>0 \quad \exists \tau>T: \quad \tau \text { есть } \varepsilon \text {-почти период } A(t) .
$$

Тогда траектория $X(t)=X\left(t, X_{0}\right)$ уравнения (1) возвращается $к$ начальному условию $X(0)=X_{0}$.

ЗАмЕчАниЕ. Поскольку уравнение (1) является линейным, возврашается величина

$$
\Xi(t)=\sup _{X_{0} \in \mathrm{SO}(n)}\left|X\left(t, X_{0}\right)-X_{0}\right|
$$

т.e.

$$
\forall \varepsilon>0 \quad \forall T>0 \quad \exists t>T: \quad \Xi(t)<\varepsilon .
$$

Отметим, что условие (2) является более слабьм, чем классическое условие (равномерной) почти периодичности (см. [6]).

Tеорема 2. Пусть матрица $A(t) \subset \operatorname{so}(n)$ условно периодически зависит от времени $t$ и $\omega_{1}, \ldots, \omega_{s}-$ базис линейно независимых над $\mathbb{Z}$ частот:

$$
\begin{gathered}
A(t)=\sum_{\left(m_{1}, \ldots, m_{s}\right) \in \mathbb{Z}^{s}} A_{m_{1}, \ldots, m_{s}} e^{2 \pi i\left(m_{1} \omega_{1}+\cdots+m_{s} \omega_{s}\right) t}, \quad A_{m_{1}, \ldots, m_{s}} \in \operatorname{so}(n), \\
K=s \sum_{\left(m_{1}, \ldots, m_{s}\right) \in \mathbb{Z}^{s}}\left|A_{m_{1}, \ldots, m_{s}}\right| \max \left\{\left|m_{1}\right|, \ldots,\left|m_{s}\right|, 1\right\}<+\infty
\end{gathered}
$$

$u d=n(n-1) / 2$. Тогда для любого $T>1$ найдется $t(T), 1 \leqslant t(T) \leqslant T$, такое, что

$$
\left|X\left(t(T), X_{0}\right)-X_{0}\right| \leqslant C(n, K, \omega)\left(\frac{\log s}{\log \log T}\right)^{1 / d}
$$

причем $t(T) \rightarrow+\infty$ при $T \rightarrow \infty$. 
ЗАмЕчАнИЕ. Очевидно, в левой части неравенства (4) можно поставить величину

$$
\Xi(t(T))=\sup _{X_{0} \in \mathrm{SO}(n)}\left|X\left(t(T), X_{0}\right)-X_{0}\right|
$$

В [7] доказано, что для почти всех (в смысле меры Лебега) наборов частот $\left(\omega_{1}, \omega_{2}\right)$ для траектории $X\left(t, X_{0}\right)$ уравнения $(1)$ с двухчастотной условно периодической матрицей $A(t)$ найдется последовательность моментов времени $t_{\nu} \rightarrow+\infty$, для которой

$$
\left|X\left(t_{\nu}, X_{0}\right)-X_{0}\right|=O\left(\left(\log t_{\nu}\right)^{-1 /(2 n+1)}\right)
$$

Относительно нижних оценок времени возврашаемости автору известен лишь классический результат, получаюшийся с помощью алгебраических чисел (см. [8]): для уравнения

$$
\dot{X}=\omega, \quad X \in \mathbb{T}^{n},
$$

в случае, ког да $\omega_{1}, \ldots, \omega_{n}$ образуют базис вещественного алгебраического поля, выполнено

$$
\left|X\left(t, X_{0}\right)-X_{0}\right| \gg t^{-1 /(n-1)}, \quad t>1 .
$$

\section{3. Вспомогательные утверждения и доказательства теорем 1 и 2.}

Лемма 1. Пусть $B_{1}, \ldots, B_{k} \in \mathrm{SO}(n), d=n(n-1) / 2$. Тогда найдутся натуральные числа $i_{1}, \ldots, i_{p}$ такие, что $1 \leqslant i_{1}<\cdots<i_{p} \leqslant k$ и $B_{i_{1}} \cdots B_{i_{p}}=E+O\left(k^{-1 / d}\right)$.

ДокАЗАТЕЛЬСтво. Применим принцип Дирихле. Рассмотрим матрицы

$$
\Omega_{j}=B_{0} B_{1} \cdots B_{j}, \quad B_{0}=E, \quad j=0, \ldots, k
$$

В силу компактности $\mathrm{SO}(n)$ можно покрыть $k$ кусочками диаметра $O\left(k^{-1 / d}\right)$. Однако, число матриц $\Omega_{j}$ в точности $k+1$. Итак,

$$
\exists j>i: \quad\left|\Omega_{j}-\Omega_{i}\right|=O\left(k^{-1 / d}\right) .
$$

Тем самьм, $\left|B_{i+1} \cdots B_{j}-E\right|=O\left(k^{-1 / d}\right)$. Лемма доказана.

Следуюшая лемма очевидна, и ее доказательство мы не приводим.

Лемма 2. Пусть $Z(t)$ и $Y(t)$-соответственно решения систем

$$
\dot{Z}=A(t) Z, \quad \dot{Y}=B(t) Y,
$$

где $A(t), B(t) \subset \mathrm{so}(n)$, с начальным условием $Z(0)=Y(0) \in \mathrm{SO}(n)$. Пусть для любого $t$ выполнено $|A(t)-B(t)|<\varepsilon$. Тогда при любом $t \in[0,1 / \varepsilon]$ имеем $|Z(t)-Y(t)| \ll \varepsilon t$. 
ДоКАЗАТЕЛЬСТво тЕОРЕмЫ 1 . Ограничимся случаем $X_{0}=E$ (единичная матрица). Зафиксируем $T$. По $\varepsilon_{1}=\varepsilon$ согласно условию (2) выберем $\tau_{1}>T$, которое является $\varepsilon_{1}$-почти периодом $A(t)$. Возьмем $\varepsilon_{2}=\varepsilon_{1} / \tau_{1}$ и выберем по нему $\tau_{2}>T$, которое является $\varepsilon_{2}$-почти периодом $A(t)$. Выполним эту процедуру $k$ раз, пока по $\varepsilon_{k}=\varepsilon_{1} /\left(\tau_{1}+\cdots+\tau_{k-1}\right)$ не определится $\tau_{k}>T$, являющееся $\varepsilon_{k}$-почти периодом $A(t)$. Рассмотрим матрицы $X\left(\tau_{1}\right), \ldots, X\left(\tau_{k}\right) \in \mathrm{SO}(n)$ и применим к ним лемму 1. Получим

$$
X\left(\tau_{i_{1}}\right) \cdots X\left(\tau_{i_{p}}\right)=E+O\left(k^{-1 / d}\right) .
$$

Оценим разность $X\left(\tau_{i_{p}}+\cdots+\tau_{i_{1}}\right)-X\left(\tau_{i_{1}}\right) \cdots X\left(\tau_{i_{p}}\right)$. Так как $\tau_{i}$ является $\varepsilon_{i}$-почти периодом, имеет место $\left|A\left(t+\tau_{i}\right)-A(t)\right|<\varepsilon_{i}$ для любого $t$. Рассмотрим системы уравнений с начальньми условиями

$$
\begin{gathered}
\dot{Y}=A\left(t+\tau_{i_{\nu+1}}\right) Y, \quad Y(0)=X\left(\tau_{i_{\nu+1}}\right), \\
\dot{Z}=A(t) Z, \quad Z(0)=X\left(\tau_{i_{\nu+1}}\right) .
\end{gathered}
$$

Для системы (5) $Y(t)=X\left(\tau_{i_{\nu+1}}+t\right)$, а для (6) $Z(t)=X(t) X\left(\tau_{i_{\nu+1}}\right)$. По лемме 2 имеем

$$
X\left(\tau_{i_{\nu+1}}+t\right)-X(t) X\left(\tau_{i_{\nu+1}}\right)=O\left(\varepsilon_{i_{\nu+1}} t\right) .
$$

Итак,

$$
\begin{aligned}
X\left(\tau_{i_{p}}+\cdots+\tau_{i_{1}}\right) & =X\left(\tau_{i_{p-1}}+\cdots+\tau_{i_{1}}\right) X\left(\tau_{i_{p}}\right)+O\left(\varepsilon_{i_{p}}\left(\tau_{i_{p-1}}+\cdots+\tau_{i_{1}}\right)\right)=\cdots \\
& =X\left(\tau_{i_{1}}\right) \cdots X\left(\tau_{i_{p}}\right)+O(k \varepsilon) .
\end{aligned}
$$

Полагая $k=\left[\varepsilon^{-d /(d+1)}\right]$ имеем $T^{*}=\tau_{i_{p}}+\cdots+\tau_{i_{1}}>T$ и $X\left(T^{*}\right)=E+O\left(\varepsilon^{1 /(d+1)}\right)$. Теорема 1 доказана.

ДОКАЗАТЕЛЬСТВО ТЕОРЕМЫ 2. ПоЛоЖим

$$
\varepsilon=\gamma\left(\frac{\log s}{\log \log T}\right)^{(d+1) / d}, \quad k=\left[\varepsilon^{-d /(d+1)}\right],
$$

$\gamma$ - достаточно малая положительная константа. Применяя теорему Дирихле о совместных приближениях [8], получим для $\varepsilon_{1}=\varepsilon$ натуральное $\tau_{1}$ такое, что

$$
\max _{j=1, \ldots, s-1}\left\|\frac{\tau_{1} \omega_{j}}{\omega_{s}}\right\| \leqslant \varepsilon_{1}, \quad \tau_{1} \leqslant \varepsilon_{1}^{-s+1} .
$$

Определим индуктивно $\varepsilon_{\mu}, \tau_{\mu}, \mu=1, \ldots, k$, так, что

$$
\varepsilon_{\mu+1}=\frac{\varepsilon}{\tau_{1}+\cdots+\tau_{\mu}}, \quad \max _{j=1, \ldots, s-1}\left\|\frac{\tau_{\mu} \omega_{j}}{\omega_{s}}\right\| \leqslant \varepsilon_{\mu}, \quad \tau_{\mu} \leqslant \varepsilon_{\mu}^{-s+1} .
$$

В силу условия (3) каждое из чисел $\tau_{\mu} / \omega_{s}$ является $K \varepsilon_{\mu}$-почти периодом $A(t)$. В силу иррациональности $\omega_{j} / \omega_{s}$ выполнено $\min \tau_{\mu} \rightarrow+\infty, T \rightarrow+\infty$. Рассуждения, применявшиеся в доказательстве теоремы 1 , позволяют утверждать, что существует момент времени $T^{*}=t(T)$ такой, что

$$
\begin{gathered}
\omega_{s}^{-1} \min _{\mu} \tau_{\mu} \leqslant T^{*} \leqslant \omega_{s}^{-1}\left(\tau_{1}+\cdots+\tau_{k}\right), \\
\left|X\left(T^{*}, X_{0}\right)-X_{0}\right| \leqslant \gamma_{1} k \varepsilon \leqslant \gamma_{2}\left(\frac{\log s}{\log \log T}\right)^{1 / d},
\end{gathered}
$$


где $\gamma_{l}=\gamma_{l}(n, K, \omega, \gamma)>0$. При достаточно малом $\gamma$

$$
\begin{aligned}
\max _{1 \leqslant j \leqslant \mu} \tau_{j} & \leqslant \varepsilon_{\mu}^{-s} \leqslant\left(\frac{\varepsilon}{\tau_{1}+\cdots+\tau_{\mu-1}}\right)^{-s} \\
& \leqslant\left(\frac{k}{\varepsilon}\right)^{s}\left(\max _{1 \leqslant j \leqslant \mu-1} \tau_{j}\right)^{s} \leqslant \cdots \leqslant\left(\frac{k}{\varepsilon}\right)^{(s+1)^{k}} \leqslant T \omega_{s} .
\end{aligned}
$$

Теперь (7) можно переписать в виде

$$
\omega_{s}^{-1} \min _{\mu} \tau_{\mu} \leqslant T^{*} \leqslant T
$$

Соотношения (8) и (9) доказьвают теорему 2.

4. Интегралы от условно периодических функций. Пусть $f: \mathbb{T}^{s} \rightarrow \mathbb{R}$ - функция, заданная абсолютно сходящимся рядом Фурье:

$$
f\left(x_{1}, \ldots, x_{s}\right)=\sum_{\substack{m \in \mathbb{Z}^{s} \\ m \neq 0}} f_{m_{1}, \ldots, m_{s}} e^{2 \pi i\left(m_{1} x_{1}+\cdots+m_{s} x_{s}\right)},
$$

частоты $\omega_{1}, \ldots, \omega_{s} \in \mathbb{R}$ линейно независимы над кольцом $\mathbb{Z}$ и $\varphi=\left(\varphi_{1}, \ldots, \varphi_{s}\right) \in \mathbb{R}^{s}$. Рассмотрим интеграл

$$
I_{f, \omega}(t, \tau, \varphi)=\int_{t}^{t+\tau} f\left(\omega_{1} \xi+\varphi_{1}, \ldots, \omega_{s} \xi+\varphi_{s}\right) d \xi
$$

Лемма 3. Для любого вещественного $\tau$ выполнено равенство

$$
\inf _{t \in \mathbb{R}}\left|I_{f, \omega}(t, \tau, \varphi)\right|=\inf _{\varphi \in \mathbb{T}^{s}}\left|I_{f, \omega}(t, \tau, \varphi)\right|=0
$$

ДокАЗАТЕЛЬСтво. Определим

$$
g\left(x_{1}, \ldots, x_{s}\right)=\sum_{\substack{m \in \mathbb{Z}^{s} \\ m \neq 0}} f_{m} \frac{e^{2 \pi i\left(m_{1} \omega_{1}+\cdots+m_{s} \omega_{s}\right) \tau}-1}{2 \pi i\left(m_{1} \omega_{1}+\cdots+m_{s} \omega_{s}\right)} e^{2 \pi i\left(m_{1} x_{1}+\cdots+m_{s} x_{s}\right)}
$$

(ряд сходится абсолютно). Отсюда видно, что $I_{f, \omega}(t, \tau, \varphi)=g\left(\omega_{1} t+\varphi_{1}, \ldots, \omega_{s} t+\varphi_{s}\right)$, т.е. интеграл $I_{f, \omega}(t, \tau, \varphi)$ является условно периодической функцией от $t$. Теперь лемма 3 является следствием всюду плотности вполне иррациональной обмотки тора.

Совершенно аналогично доказьвается

\section{Лемма 4. Имеют место равенства}

$$
\sup _{t \in \mathbb{R}}\left|I_{f, \omega}(t, \tau, \varphi)\right|=\sup _{\varphi \in \mathbb{T}^{s}}\left|I_{f, \omega}(t, \tau, \varphi)\right|=\sup _{\varphi \in \mathbb{T}^{s}}\left|I_{f, \omega}(0, \tau, \varphi)\right| \text {. }
$$

В работе [9] на основании свойств сингулярных по Хинчину систем (см. [10], [11]) доказана 
Лемма 5. Пусть $s \geqslant 3$. Тогда для любой положительнозначной функиии $\Psi(y)$, сколь угодно быстро убывающей $\kappa 0, u$ для любой положительнозначной функиии $\psi(y)$ такой, что $\psi(y)=o(y), y \rightarrow+\infty$, найдется набор линейно независимых над $\mathbb{Z}$ частот $\omega_{1}, \ldots, \omega_{s}$ со следуюшим свойством:

для любой функиии $f$, коэффициенты Фурье которой допускают оценку

$$
\left|f_{m_{1}, \ldots, m_{s}}\right| \geqslant \Psi\left(\max _{j}\left|m_{j}\right|\right) \quad \text { npu } \quad \max _{j}\left|m_{j}\right| \geqslant M_{0}=M_{0}[f],
$$

имеет место соотношение

$$
\exists \tau_{0}=\tau_{0}\left[\Psi, \psi, M_{0}\right]: \quad \forall \tau \geqslant \tau_{0} \quad \sup _{\varphi \in \mathbb{T}^{s}}\left|I_{f, \omega}(0, \tau, \varphi)\right| \geqslant \psi(\tau) .
$$

Из лемм 3-5 и соображений непрерьвности вытекает

ТЕОрема 3. Пусть $s \geqslant 3$. Тогда для любой положительнозначной функиии $\Psi(y)$ найдется набор линейно независимых над $\mathbb{Z}$ частот $\omega_{1}, \ldots, \omega_{s}$ со следующим свойством:

для любой функиии $f$, коэффициенты Фурье которой допускают оченку

$$
\left|f_{m_{1}, \ldots, m_{s}}\right| \geqslant \Psi\left(\max _{j}\left|m_{j}\right|\right) \quad n p u \quad \max _{j}\left|m_{j}\right| \geqslant M_{0}=M_{0}[f]
$$

имеет место соотношение

$$
\exists \tau_{0}=\tau_{0}\left[\Psi, M_{0}\right]: \quad \forall \tau \geqslant \tau_{0} \quad \forall \varphi \in \mathbb{T}^{s} \quad \sup _{t \in \mathbb{R}}\left\|I_{f, \omega}(t, \tau, \varphi)\right\|=\frac{1}{2}
$$

( $\mid \|$ - $\|$-расстояние до блиэсайшего челого).

Отметим, что при $s=2$ теорема 3 неверна: в работах [12], [13] для любой достаточно гладкой функции $f\left(x_{1}, x_{2}\right)$ и для любых несоизмеримых частот $\omega_{1}, \omega_{2}$ доказано выполнение условия

$$
\forall \varepsilon>0 \quad \forall \tau^{*} \exists \tau>\tau^{*}: \quad \sup _{t \in \mathbb{R}}\left|I_{f, \omega}(t, \tau, \varphi)\right|<\varepsilon,
$$

т.е. возвращаемость величины $\sup _{t \in \mathbb{R}}\left|I_{f, \omega}(t, \tau, \varphi)\right|$ при $\tau \rightarrow \infty$. Тем не менее, мы можем сформулировать результат об аномальном поведении рассматриваемого интеграла и при $s=2$. Этот результат связан с наличием частот $\omega_{1}, \omega_{2}$, отношение которых допускает аномально хорошие рациональные приближения. Его доказательство фактически проведено в [14, теорема 1 , случай Б].

ТЕОрема 4. Пусть $s=2$. Тогда для любой положительнозначной функиии $\Psi(y)$ и любой полоэительнозначной функиии $\psi(y)$, монотонно стремящейся $\kappa 0$ при $y \rightarrow \infty$, найдется набор линейно независимых над $\mathbb{Z}$ частот $\omega_{1}, \omega_{2}$ со следующим свойством:

для любой функиии $f$, коэффициенты Фурье которой допускают оченку

$$
\left|f_{m_{1}, m_{2}}\right| \geqslant \Psi\left(\max _{j}\left|m_{j}\right|\right) \quad n p u \quad \max _{j}\left|m_{j}\right| \geqslant M_{0}=M_{0}[f],
$$

для некоторой последовательности моментов времени $T_{\nu} \rightarrow \infty$ имеет место соотношение

$$
\min _{1 \leqslant \tau \leqslant T_{\nu}} \sup _{t \in \mathbb{R}}\left\|I_{f, \omega}(t, \tau, \varphi)\right\| \geqslant \psi\left(T_{\nu}\right) .
$$

Отметим, что левая часть этой формулы на самом деле не зависит от $\varphi$. 
5. Равномерная возвращаемость. Контрпример. Вернемся к уравнению (1). Как отмечалось в п. 2, возврашаемость величины $\Xi(t)$ равносильна возврашаемости отдельной траектории. Далее мы будем рассматривать другую характеристику равномерной возвращаемости и для уравнения (1) определим величину

$$
\Lambda(\tau)=\sup _{t \in \mathbb{R}} \sup _{X_{0} \in \mathrm{SO}(n)}\left|X\left(t+\tau, X_{0}\right)-X\left(t, X_{0}\right)\right| .
$$

Ниже приведен пример системы уравнений, для которых величина $\Lambda(\tau)$ не возвращается.

На окружности $\mathrm{SO}(2)$, отнесенной к координате $z(\bmod 1)$, рассмотрим уравнение $\mathrm{c}$ условно периодическими коэффициентами

$$
\dot{X}=A(t) X, \quad X=\left(\begin{array}{cc}
\cos 2 \pi z & \sin 2 \pi z \\
-\sin 2 \pi z & \cos 2 \pi z
\end{array}\right), \quad A(t)=\left(\begin{array}{cc}
0 & a(t) \\
-a(t) & 0
\end{array}\right),
$$

где

$$
a(t)=f\left(\omega_{1} t, \ldots, \omega_{s} t\right)=\sum_{\substack{m \in \mathbb{Z}^{s} \\ m \neq 0}} f_{m_{1}, \ldots, m_{s}} e^{2 \pi i\left(\omega_{1} x_{1}+\cdots+\omega_{s} x_{s}\right) t} .
$$

Поскольку решение рассматриваемого уравнения явным образом записывается с помощью интеграла $I_{f, \omega}(t, \tau, \varphi)$, теоремы 3 и 4 принимают следующий вид.

ТЕОРема 5. Пусть $s \geqslant 3$. Тогда для любой положительнозначной функиии $\Psi(y)$ найдется набор линейно независимых над $\mathbb{Z}$ частот $\omega_{1}, \ldots, \omega_{s}$ со следующим свойством:

для любой функиии $f$, коэффичиенты Фурье которой допускают оченку

$$
\left|f_{m_{1}, \ldots, m_{s}}\right| \geqslant \Psi\left(\max _{j}\left|m_{j}\right|\right) \quad n p u \quad \max _{j}\left|m_{j}\right| \geqslant M_{0}=M_{0}[f],
$$

для уравнения (10)

$$
\Lambda(\tau)=\max _{\xi, \eta \in \mathrm{SO}(2)}|\xi-\eta|>0 \quad n p u \quad \tau \geqslant \tau_{0},
$$

т.е. величина $\Lambda(\tau)$ не возвращается.

ТЕОРема 6. Пусть $s=2$. Тогда для любой положительнозначной функиии $\Psi(y)$ и любой положительнозначной функиии $\psi(y)$, монотонно стремящейся $\kappa 0$ nри $y \rightarrow \infty$, найдется набор линейно независимых над $\mathbb{Z}$ частот $\omega_{1}, \omega_{2}$ со следующим свойством:

для любой функиии $f$, коэффичиенты Фурье которой допускают оченку

$$
\left|f_{m_{1}, m_{2}}\right| \geqslant \Psi\left(\max _{j}\left|m_{j}\right|\right) \quad \text { npu } \max _{j}\left|m_{j}\right| \geqslant M_{0}=M_{0}[f],
$$

для некоторой последовательности моментов времени $T_{\nu} \rightarrow \infty$ имеет место соотношение

$$
\min _{1 \leqslant \tau \leqslant T_{\nu}} \Lambda(\tau) \geqslant \psi\left(T_{\nu}\right) .
$$

Теоремы 5, 6 можно рассматривать как пример того, что "интеграл" почти периодической функции (решение уравнения (10)) ограничен, но не является почти периодической функцией. 
6. Возвращаемость и приводимость. Существуют различные определения приводимости (см. [15]-[19]). Будем говорить, что уравнение (1) приводимо, если существует замена переменных $X=B(t) Y$ с невырожденной условно периодической матрицей $B(t), B(0)=E$, с базисом частот $\omega_{1}^{*}, \ldots, \omega_{\sigma}^{*}$ такая, что после замены переменных уравнение (1) принимает вид

$$
\dot{Y}=D Y
$$

где $D$ - постоянная матрица. Поскольку исходное уравнение рассматривается на компактной группе Ли $\mathrm{SO}(n)$, справедливы следующие наблюдения.

ЛЕмма 6. В случае приводимости уравнения (11) выполнено $D \in \operatorname{so}(n)$ u, следовательно, $B(t) \subset \mathrm{SO}(n)$.

Для доказательства достаточно заметить, что в силу невырожденности и условной периодичности замены матрицы $B(t)$ и $(B(t))^{-1}$ ограничены при всех $t$ и, следовательно, $e^{D t}$ ограничена при всех $t \in \mathbb{R}$.

ЛЕмма 7. Если уравнение (1) приводимо, то для любого $T>1$ найдется $t(T)$, $1 \leqslant t(T) \leqslant T$, такое, что

$$
\Lambda(t(T))=\sup _{t \in \mathbb{R}} \sup _{X_{0} \in \operatorname{SO}(n)}\left|X\left(t(T)+t, X_{0}\right)-X\left(t, X_{0}\right)\right| \leqslant \varkappa T^{-1 /(n+\sigma)},
$$

причем $t(T) \rightarrow+\infty$ при $T \rightarrow \infty$.

Лемма 7 вытекает из теоремы Дирихле о совместных приближениях [8], примененной для базиса частот $\omega_{1}^{*}, \ldots, \omega_{\sigma}^{*}$ матрицы приведения и показателей $\lambda_{1}, \ldots, \lambda_{n}$, определяемых характеристическими числами $\chi_{1}=e^{2 \pi i \lambda_{1}}, \ldots, \chi_{n}=e^{2 \pi i \lambda_{n}}$ постоянной ортогональной матрищы $e^{D}$.

ЗАмЕчаниЕ. Лемма 7 показьвает, что пример (10) со сколь угодно большим наперед заданным порядком гладкости правой части при определенном выборе частот обладает следующими свойствами:

1) для “почти всех" условно периодических матрищ $A(t)$ заданной гладкости, имеющих базис частот из теорем 4,5 , уравнение (10) неприводимо;

2) каждая траектория уравнения (10) ограничена.

7. Структура невозвращаемости. Примеры этого пункта показывают, что величина $\Lambda(\tau)$ может не возврашаться. Опишем комбинаторную структуру такой невозвращаемости. Согласно теореме 1 для матрицы $A(t)$, удовлетворяющей $(2)$, для любых $\varepsilon, T$ и каждого $\tau$ найдется момент $t^{*}(\varepsilon, T) \geqslant T$ возвращаемости траектории $X\left(t+\tau, X_{0}\right)$ в $\varepsilon$-окрестность точки $X\left(t, X_{0}\right)$. Этот момент времени имеет вид $t^{*}(\varepsilon, T)=$ $\tau_{i}+\cdots+\tau_{j}, i<j$, где величины $\tau_{i}, \ldots, \tau_{j}$ представляют собой несколько последовательно идущих чисел из набора $\tau_{1}, \ldots, \tau_{k}, k=O\left(\varepsilon^{-d}\right)$, зависящего только от $\varepsilon$ и $T$. Начало $i$ и конец $j$ выборки зависят от $t$. Кроме того, для каждого $t$ соответствующая ему выборка $\tau_{i}, \ldots, \tau_{j}$ обеспечивает возвращаемость в $2 \varepsilon$-окрестность точки $X\left(t, X_{0}\right)$ не только для самого $t$, но и для всех моментов времени в некоторой окрестности момента $t$. Следующая теорема отражает эту картину возвращаемости. 
Теорема 7. Пусть матрица $A(t)$ удовлетворяет условию (2). Тогда для любого $\varepsilon>0$ найдется число $H(\varepsilon)=O\left(\varepsilon^{-d}\right), d=n(n-1) / 2$, такое, что для любого $T$ можнно указать совокупность интервалов

$$
J^{h}(\varepsilon, T)=\bigcup_{i=-\infty}^{+\infty}\left(t_{i}(\varepsilon, T), t_{i+1}(\varepsilon, T)\right), \quad 1 \leqslant h \leqslant H(\varepsilon),
$$

таких, что

$$
\bigcup_{h=1}^{H(\varepsilon)} J^{h}(\varepsilon, T)=(-\infty,+\infty),
$$

и для каждого $h$ можнно указать момент времени $\tau_{h}=\tau_{h}(\varepsilon, T)>T$ такой, что

$$
\Lambda_{h}\left(\tau_{h}\right)=\sup _{t \in J^{h}(\varepsilon, T)} \sup _{X_{0} \in \operatorname{SO}(n)}\left|X\left(t+\tau_{h}, X_{0}\right)-X\left(t, X_{0}\right)\right|<\varepsilon .
$$

Отметим, что интересно получить точные по порядку оценки величины $H(\varepsilon)$, а также описать возможные структуры последовательности переключений величины $h$.

\section{СПИСОК ЦИТИРОВАННОЙ ЛИТЕРАТУРЫ}

[1] Пуанкаре А. Новые методы небесной механики // Избранные труды. Т. 2. М.: Наука, 1972.

[2] Мощевитин Н. Г. Об одной теореме Пуанкаре // УМН. 1998. Т. 53. №1. С. 223-224.

[3] Furstenberg H. Recurrence in Ergodic Theory and Combinatorial Number Theory. Princeton: Princeton Univ. Press, 1981.

[4] Baker R. C. Recent results on fractional parts of polynomials // Lecture Notes in Math. 1979. V. 751. P. 10-17.

[5] Boshernizan M.D. Elementary proof of Furstenberg Diophantine result // Proc. Amer. Math. Soc. 1994. V. 122. №1. P. 67-70.

[6] Левитан Б. М. Почти периодические функции. М.: ГИТТЛ, 1953.

[7] Лапин С. Э. О возвращаемости траекторий систем линейных дифференциальных уравнений с условно периодическими коэффициентами // Матем. заметки. 1993. Т. 53. №1. С. 52-56.

[8] Касселс Дж. Введение в теорию диофантовых приближений. М.: ИЛ, 1961.

[9] Мощевитин Н. Г. Распределение значений линейных функций и асимптотическое поведение траекторий некоторых динамических систем // Матем. заметки. 1995. Т. 58. № 3. С. 394-410.

[10] Khinchin A. Ya. Über eine Klasse linearer Diophantine Approximationen // Rend. Circ. Mat. Palermo. 1926. V. 50. P. 170-195.

[11] Хинчин А.Я. Регулярные системы линейных уравнений и общая задача Чебышева // Изв. АН CCCP. Сер. матем. 1950. Т. 12. С. 249-258.

[12] Козлов В. В. Финальные свойства интегралов от квазипериодических функций // Вестн. МГУ. Сер. 1. Матем., мех. 1978. № 1. С. 106-115.

[13] Козлов В. В. Методы качественного анализа в динамике твердого тела. М.: Изд-во МГУ, 1980.

[14] Козлов В. В., Мощевитин Н. Г. О диффузии в гамильтоновых системах // Вестн. МГУ. Сер. 1. Матем., мех. 1997. № 5. С. 49-52.

[15] Еругин Н.П. Приводимые системы. Тр. МИАН. Т. 13. Л.-М.: АН СССР, 1946.

[16] Арнольд В.И. Дополнительные главы теории обыкновенных дифференциальных уравнений. М.: Наука, 1978.

[17] Treshchev D. V. An estimate of irremovable nonconstant terms in the reducibility problem // Amer. Math. Soc. Transl. Ser. 2. 1995. V. 168. P. 91-128.

[18] Блинов И.Н. Прямой метод. Приводимость // Изв. АН СССР. Сер. матем. 1990. Т. 54. № 1. C. 201-212.

[19] Бронштейн И.У., Чёрный В.Ф. Линейные расширения, удовлетворяюшие условию Перрона. I // Дифференц. уравнения. 1978. Т. 14. №10. С. 1739-1751; II // Дифференц. уравнения. 1980. Т. 16. № 2. С. 201-207. 